T. Palberg

M. Würth

J. Schwarz

P. Leiderer

\section{Kinetics of crystal growth in charged colloidal suspensions}

Dr. T. Palberg (四) - M. Wǔrlh

J. Schwarz-P Leiderer

Faculty of Physics

University of Konstanz

P. O. Box $5560 \mathrm{M} 676$

78434 Konslanz, FRG
A thitract We report on the

solidification of a metastable colloidal mell of monodisperse, highly charged latex spheres. Light-scattering and video microscopy are used to study the growth velocities of crystals nucleated at the walls of the observation cell and in the bulk melt. The velocity observed for the planas (110) face of the body-centered cubic wall crystals $v_{110}$ is lound to be significantly smaller than the radial growth velocity $v_{R}$ of the homogeneously nucleated crystals of rounded polyhedric shape. Under isothermal conditions the interaction determining suspension parameters packing fraction $\Phi$ salt concentration $c$, and surface charge $Z$ were systernatically varied with high accuracy using advanoed preparation methods. Growth velocities $v_{110}$ in the $\langle 110\rangle$ direction increase over more than three orders of magnitude with increasing $\Phi$ and becreasing $c$. All data collapse on a single curve if plotted against a reduced energy density $\Pi^{*}$ between melt and fluid al melting. This master curve shows an initially linear increase and sacurates at large $\Pi^{*}$ with $v_{\infty}=9.1 \mu \mathrm{ms}^{-1}$ as the limiting velocity. It can be excellently fitted with a Wilson-Frenkel growth law yielding a conversion factor of $\mathrm{B}=6.7 k_{\mathrm{B}} T$ between $\Pi^{*}$ and the chemical potential difference $\Delta \mu$ between melt and solid. Detailcd analysis of the saturation value $v_{\infty}$ provides evidence for two different growth mechanisms operative in the solidification of colloidal crystals

Key words Charged colloids colloidal crystals - crystal growth Wilson-Frenkel-growth time-resolved static light scattering

\section{Introduction}

There are numerous examples of colloid and interface systems forming supramolecular order ranging from equilibrium lamellar and cubic phases in microemulsion to shear-induced ordering of latex spheres, from nematic to cholesteric and from ringing gels to opals. Many of these systems offer extremely rich phase diagrams with a large number of parameters. Moreover, they often reveal complicated coupled dynamics on a multitude of time scales
[1]. Investigations of the phase transition kinetics prove extremely difficult due 10 the great complexity of the systcms, yet adding another facet to the fascinating and useful interdisciplinary field of colloid science. The stracegy 10 solve this problem in the long run will lie in resorting 10 comparably simple systerns and to concepts from condensed matter physios first and then gradually introducing larger numbers of system specific boundary conditions.

Solidification of materials in general is of great imporarce in the processing of materials and has a long bistory of investigation. A number of semiempirical theories of 
nucleation and growth have already been deviloped in the first part of this century which capture the basic features of the first order freezing Iransition $[2,3]$. Their quantitative experimental verification and the development of corresponding microscopic models for the relevant kinetic processes face two major problems even in the case of aloraic or molecular systems with only a small number of internal degrees of freedom. The relevant length scales are accessible only with high technical effort. Moreover direct observation is difticult due to the high rates of nucleation and high velocities of growth. As a consequence experimental data of both high spatial and temporal resolution are rare. In addition, poorly defined or changing experimental conditions often restrict quantitalive comparisons to the above-mentioned theories. Limited thermal diffusion, for example. often prevents the development of sufficiently stationary condifions during and after an applied temperature quench or the release of latent heat.

Colloidal systems, on the other hand, may be isothermally shear molten due to the low particle density of some $10^{12}$ to $10^{14} \mathrm{~cm}^{-3}$ and the presence of the suspending fluid acting as a heat barh [4]. This allows for the formation of sufficiently stationary states after quasi instantaneous quenches. Solidifying samples may be prepared and observed by opeical techniques under conditions very close to those defined by the assumptions of the above mentioned theories [5]. Rigid spherical particles suspended in a homogeneous medium would provide a particularly simple example of solidifying colloidal systems. Freezing and melting are located at packing fractions of $\Phi_{t}=0.494$ and $\Phi_{m}=0.521$, respectively [6]. Suspensions with electrostatic stabilization solidify at considerably smaller packing fraction depending on the particle charge and the amount of scrcening electrolyte $c[7-10]$. The presence of the viscous suspending nedium shifts the relevant time scales to the range between milliseconds and hours. This facilitates observations in real and in reciprocal space with high temporal and spatial resolution. Finally, as a point imporlant for systematic and quantitative measurements, one can precisely adjust the intcraction between the suspended particles while it is well described by theoretical expressions [11].

These colloid-specific advantages have led to a strong interest in the solidification dynamics of colloidal crystals and a considerable number of papers exist both on the nucleation and the growth from the melastable shear melt $[4,11-18]$. Still, the body of systematic data is yet 100 small for rigorous and comprehensive comparisons to the aforementioned theories or to recent computer simulations [19].

We here summarize some of our recent results on the growh kinetics observed during the solidification of metastable melts of highly charged latex spheres. W'e report systematic studies under variation of the packing fraction, the strength and the range of the Yukawa interaction. We focus on both the presentation of bigh precision data and their detailed quantitative analysis. The paper is organized as follows. The second section gives a short description of the experimental system and the methods used in preparing well-defined shear melts. We then concentrate on the growth velocities to give unequivocal evidence for Wilson Frenkel growth in bighly charged colloidal suspensions. Finally, the structure of the solid-melt interface is discussed in some detail in the fourth section.

\section{Expormental}

Two kinds of particles were used in this study which have been characterized extensively by light scattering and otber methods [20]. In particular, we used commercially available polystyrene latex spheres (Seradyn, U.S.A. Lot Nos. 2010M9R and 201 IM9R) of equal hydrodynamic radius $a_{h}=51 \mathrm{~nm}$ (dynamic light scattering), but different surface group numbers $N$. At completely deionized conditions the renormalized or effective charge numbers differ by some $15 \%$. The pair interaction potential is well de. scribed using either the renormalization procedures or the modified DLVO approximation (MDA), as has been tested by shear modulus titration and also by dynamical criteria [20-22]. A compilation of particle and suspension data is given in Table 1.

The monodisperse particles crystallize into a bodycentered cubic (bec) lattice if the packing fraction is above $\phi=0.002$ and the concentration of added salt $c$ is in the sub-micromolar range [20]. To adjust the suspension parameters $\Phi$ and $c$ with errors below $1 \%$ and $2 \%$, respectively, a recently developed advanced preparation procedure is used [23]. The suspension flows through the observation cell under a hydrostatic pressure difference between two reservoirs of adjustable height difference. It is peristaltically pumped back to the upper reservoir through an inert Teflon tubing system connecting a separate ion exchange chamber, a conductivity measurement to control the salt concentration, and a Debye-Scherrer-like setup to determine the packing fraction via static light scaltering. The ion exchange chamber may be bypassed, and salt or further suspension added under inert gas atmosphere to the reservoirs. This technique allows for fast and reproducible preparation of gradient free samples with well-characterized suspension parameters.

During preparation the suspension is in a shear molten state which readily solidifies after termination of flow by closing the two electromagnetic valves at the cell in- and outlet. Using flat shear cells with a ratio between height 
Table 1 Compilation of experimental data for the two samples. The range of variation of suspension parameter is indicaled where approprate.

\begin{tabular}{|c|c|c|c|c|c|c|}
\hline Sample & $\begin{array}{l}\text { Diameter } \\
\sigma[\mathrm{nm}]\end{array}$ & $\begin{array}{l}\text { Packing } \\
\text { fraction } \\
\Phi\end{array}$ & $\begin{array}{l}\text { Salt } \\
\text { concentration } \\
c_{x}\left[\mu \mathrm{mol} l^{-1}\right]\end{array}$ & $\begin{array}{l}\text { Surlace group } \\
\text { number } \\
N\end{array}$ & $\begin{array}{l}\text { Charge } \\
\text { number } \\
Z\end{array}$ & $\begin{array}{l}\text { Renormalized } \\
\text { chargc } \\
\text { X,pac }\end{array}$ \\
\hline $\begin{array}{l}A \\
B\end{array}$ & $\begin{array}{l}102 \\
102\end{array}$ & $\leq 0.0024$ & $\begin{array}{l}0-2 \\
0-2\end{array}$ & $\begin{array}{r}1200 \\
950\end{array}$ & $\frac{-}{580}$ & $\begin{array}{l}450 \\
395\end{array}$ \\
\hline
\end{tabular}

and depth of $K>10$ the growth of wall nucleated crystals is usually completed before significant disturbance by homogeneous nucleation processes occur. Furthermore, a high shear rate during preparation significantly increases the induction period for the formation of critical nuclei. On the other hand, homogeneous nucleation and radial growth are the predominant mechanisms of solidification in cells with small $K$ and after lower preparation shear rates. In both cases, however, the variation of shear parameters during the preparation did not lead to an observable change in the growth velocities.

We measured the propagation velocity of a planas (110) face of a bcc crystal nucleated at the cell wall against the metastable melt. The image of a laser beam crossing

Flg. 1 Comparison of the velocities of radial growh (o) to those measured for a planar (110) interface (0) in sample A. We show the evolution of the crystal radii, respective the wall crystal thickness, as a function of time. Growth is strictly linear in both cases. At $\Phi=0.0022$ and $c=0.5 \mu \mathrm{mol}]^{-1}$ the radial growth velocity $v_{n}=9.6 \mathrm{mms}^{-1}$ is considerably larger than for the planar interface $v_{110}=8.4 \mu \mathrm{ms}^{-1}$. Note that the wall crystal thickness quasi instantaocously jumps to a finite value. This might be caused by the registering of shear stabilized layers adjacent to the cell wall during preparation. Note also the the lag time observed for homogeneous nucleation which also is observed to depend on the shearing conditions during preparation. The growth velocities, however, are found to be independent of the sample history in both cases



the cell is monitored with a long distance telescopic microscope (QM1, Questar, NL) under an angle of observation not fulfilling the Bragg condition. This angle is chosen such that the structure factor $S(k)$ of the fluid phase has a significantly larger value than that of the crystalline phase. In the resulting video frames regions of largely different intensities are clearly visible. Bach of these frames is further analyzed using image processing (Optimas, Stemmer, Germany) directly yielding the temporal evolution of the spatial intensity distribution and thus the velocity of growth.

It is instructive to qualitatively compare this growth velocity in $\langle 110\rangle$ direction to the radial growth velocity of homogeneously nucleated crystals. For the latter measurements the cell is mounted on the stage of a polarization microscope (Laborlux 12, Leitz Germany) and the shape and size of crystals is directly observed. In Fig. 1, we show two data sets measured in different cells, but on the same suspension. Growth of the wall crystal starts immediately after stopping the shear, but is significantly slower. In both cases the growth is strictly linear in time. This was ob. served for all experimental conditions resulting in complete solidification. In agreement with recently reported studies on growth instabilities [14] nonlinear growth was observed only in the coexistence region of the phase diagram.

\section{Wheon-freatkol growth}

In their pioneering work, Aastuen et al. were the first 10 observe a monotonous increase in the radial growth velacities $v_{k}$ as a function of the packing fraction $\Phi$ in a system of highly charged colloidal spheres [12, 13]. In weakly charged systems the growth rate of the linear dimension of crystallites was reported to be fairly independent of $\Phi[15]$, whereas in hard sphere systems it shows a pronounced maximum in the upper coexistence range and decays 10 practically zero as the glass transition is approached [17]. In the latter case density fluctuations were observed to give rise to a $t^{1 / 2}$ behavior as a function of time $t$. 
In this study, we vary the particle charge and the concentration of screening electrolyte in addition to the packing fraction. In Figs. 2 and 3, we show the results for the propagation velocities of the planar boe (110) faces obtained for our system of wall nucieated crystals. The growth velocity $v_{110}$ increases over more than (wo orders of magnitude, if $\phi$ is increased above melting. and saturates at a value of approximately $9 \mu \mathrm{ms}^{-1}$. Similar results are observed for the salt-dependent growth velocities of the (wo differently charged samples $A$ and $B$. The phase boundary for the higher charged sample $A\left(Z_{\mathrm{PAC}}=450\right)$ is situated at significantly higher salt concentrations even though the packing fraction of that sample $(\Phi=0.0022)$ is somewhat lower than for sample $B \quad\left(Z_{\mathrm{PBC}}=395\right.$; $\Phi=0.003$ ). The limiting velocities, however, are practically identical. Data measured at equilibrium coexistence are given by the stars and will not be included in further analysis.

We further proceed much in the spirit of Aastuen et al. [12]. They suggested a Wilson-Frenkel growh law to interpret their data:

$v=v_{\infty}\left[1-\exp \left(-\frac{\Delta \mu}{k_{B} T}\right)\right]$

Fig. 2 Propagation velocity $\pi_{110}$ of the planar $(110)$ interface for a completely deionized sample $A\left(Z_{\mathrm{PBC}}=450\right)$ as a function of increasiog packing fraction $\$$. The stars denote dala recorded in the acxistence region of the phase diagram

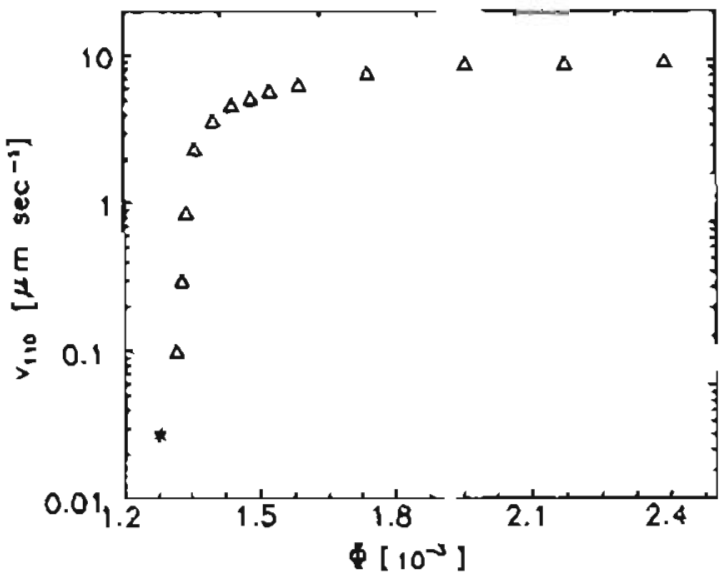

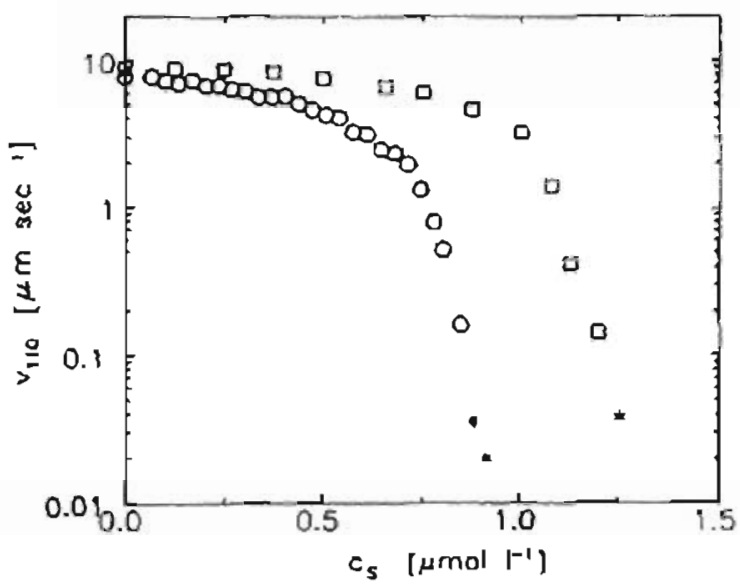

Fig. 3 Propagation velocity ${ }_{10}$ of the planar ( 110 ) interface for $(0)$ sample $A\left(Z_{\mathrm{phC}}=450, \Phi=0.0022\right)$ and (c) sample $\mathrm{B}\left(Z_{\mathrm{plc}}=395\right.$ : $\Phi=0.003$ ) as a function of increasin? salt concentration. Stars again denoie data recordod in the cocxistence region of the phase diagram. Note the coincidence of the velocities at deionized conditions for the iwo difirently charged sampis; of equal diameter $\sigma=11$ :. $\mathrm{um}$

Here, $v_{\infty}$ is a limiting velocity determined by the selfdiffusion of particles of the melt to theis target place in the crystal lattice. $k_{B} T$ denotes the thermal energy and the difference in chemical potential $\Delta \mu$ between metastable melt and solid was approximated by the reduced difference in particle densitier $A \mu=B\left(\rho-\rho_{m}\right) / \rho_{m}$, where $m$ denotes the value at melting. $A$ fit of Eq. (1) was compatible with their experimental data, a quantitative verification of the initial lineas increase and the saturation at large $\Delta \mu$ was, however, still missing. Though appropriate for their study in dependence on $\phi=3 \rho / 4 \pi a^{3}$, this approximation does not capture variations in the other suspension paramelers. We will therefore derive a reduced energy densily difference suited for particles interacting via a Yukawa-type potential formulated within the recently tested modified DLVO approximation $[21,22]$ :

$V(r)=Z_{\mathrm{PBC}}^{2} \lambda_{\mathrm{B}}\left(\frac{e^{+\kappa s}}{1+\kappa a}\right)^{2} \frac{e^{-x r}}{r}$

with the screening parameter $\kappa$ given as:

$\kappa^{2}=\lambda_{B}\left(\left|Z_{P B C}\right| \rho+2000 N_{A} c \kappa^{2}\right)$.

\footnotetext{
We note, that our present analysis neglects several points, needed for a comprehensive understanding. Most imponantly 1 he choice of $n_{m}$ as reference point may seem somewhat arbitrary. However, if we use $\Pi^{*}=\pi-\Pi_{t} / \Pi_{\mathrm{r}}$. the correct value at freesing. and fit Eq. (1) lo the data points taken above meleing only, the extrapolation of the wilson-Frenkel curve to zero growth velocily yiclds $\Pi^{\circ}=\Pi_{\mathrm{m}}$. This is equivalent to a horizontal shift of the master curve by $\Pi_{\mathrm{n}}$ in a plot of $v_{110}$ vs. $\Pi^{* *}$. Correspondingly in our Fig. 4 the data points at coexistence are at negative $\Pi^{*}$. Ao improved description therelore should consider two here, which are important at low $\Pi^{\circ}$, ie where energetic differences are small. Moreover, at coexistence the erystallization scenario might be signifieanlly different, since the development of a density difierence belween fluid and coexisting crystal should aclually be governed by collective diffusion. Consequently. the Wilson Frenkel prelactor will not remain constant. The data points taken al coexistence are therelore nol deseribed by Eq. (1) with $v_{3}=$ coust., and $\Pi^{\bullet}$ defined by energetic criterid alone. Furcher work in this direction is currencly in progress.
} 
where $\lambda_{\mathrm{B}}=e^{2} / 4 \pi \varepsilon \varepsilon_{0} k_{\mathrm{B}} T$ is the Bjerrum length which is about $0.7 \mathrm{~nm}$ in watcr. $Z_{\mathrm{PHC}}$ is the renormalized charge number derived from the barc charge number $Z[9,22,23]$.

We define a quantity $\Pi=\rho V(r)$ which formally has the dimension of an osmolic pressure. The data are then plotted against the reduced energy density difference between the metastable melt and the thuid at melting $\Pi^{*}=\left(\Pi-\Pi_{m}\right) / \Pi_{m}$. As is shown in Fig. 4, all three experimental series collapse on a single curve.

We fit the master curve with Eq. (1) and obtain a value of $v_{\infty}-9.1 \mu \mathrm{ms}^{-1}$ for the limiting velocity. The fit to the data is exiremely good for $\dot{\Pi}>\Pi_{\mathrm{m}}$ and we consider this the first unambigous experimental verification of WilsonFrenkel growth in colloidal systems. Moreover, the quality of the data allows the derivation of a proportionality factor between $\Pi^{*}$ and the chemical potential difference as $\Delta \mu=B \Pi^{*}$ with $B=6.7 k_{\mathrm{B}} T$. Il provides a quick but reasonably accurate estimate for $\Delta \mu$ given the suspension parameters and one point of the melting line.

The prefactor $v_{\infty}$ describes a limiting velocity. It is considerably lower than the limiting radial growth velocity observed by Aastuen et al. for only slightly smaller particles [12]. This is, however, consistent with the differences between $v_{110}$ and $v_{k}$ shown in Fig. 1. To explain this difference, we suggest the presence of alternative growth mechanisms.

In recent computer simulations on the growth of planar (100) and (111) faces of face-centered cubic (foc) Lennard-Jones crystals it was observed that two different mechanisms were operative [19]. For the (100) face growth was limited by the thermal velocity of the particles. It has

Fig. 4 Propagation velocity $v_{110}$ for all three series plotted versus the reduoed energy density difference $\Pi^{\circ}$. Symbols are as in Figs. 2 and 3. All data collapse on a single curve. The solid line is the best fit of Eq. (1) 10 the experimental data using $v_{110}=9.1 \mu \mathrm{ms}^{-1}$ and $\mathrm{B}=6.7 \mathrm{k}_{\mathrm{B}} \mathrm{T}$

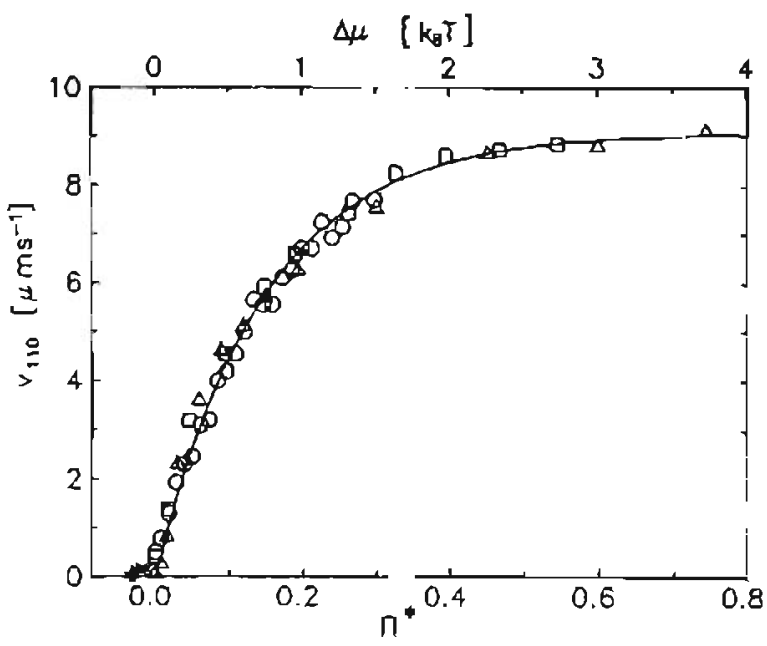

been suggested to use the diffusional transport of particles towards the rough interface of homogeneously nucleatcd crystals as the corresponding mechanism in colloidal systems [12]. Assuming bulk diflusion, we set $v_{\infty}=6 D_{1} 1 /$ $\left.\left(d_{1}, 0\right)\right)^{2}$, where $D_{\mathrm{l}}=0.1 D_{\mathrm{s}}$ is the long time self-diflusion coefficient which is about of one-tenth of the short-lime self-diffusion coefficient $D_{\mathrm{S}}$ throughout the metastable melt. $l$ is the interlacial thickness and $\lambda=0.39$ is the average relative distance of homogeneously discributed points from the center of a sphere of diameter $d_{110}$. Equat. ing $/$ to one lattice spacing $d_{110}$ leads to a limiting velocity of $v_{\infty}=33 \mu \mathrm{ms}^{-1}$, much larger than the values measured here on $v_{110}$ or $v_{R}$. Increasing / yields even larger values. Unfortunately, we do not have enough data on the radial growth velocities of our system to safely extract a limiting radial velocity. We note, however, that using our analysis on the data of Aastuen their limiting radial velocity is quantitatively reproduced. This strongly supports bulk long-time self-diffusion of particles towards their target places witbin a rough interface of one layer thickness to be the microscopic mechanism of growth limitation in the case of rounded polyhedric crystals formed after homogeneous nucleation in the bulk.

Since this mechanism has to be excluded for the growth of our bec (110) face, we have to check for alternatives. For the (111) face of the Lennard Jones crystals the simulations [19] shows that growth is limited by a reaction-like process operative on interfaces with more than one possibility of arranging the next layer. In the fcc system this is the stacking sequence. For our bcc (110) lace it is the possible formation of twins. At such an interface the melt first acquires a layered strcture, which then shows in-plane crystalline ordering and finally registers on the underlying solid layer. This last step is velocity determining. In a recent experimental study on the oucleation of fcc colloidal crystals in the vicinity of a cell wall, Grier and Murray observed a similar three-step mechanism through the evolution of a sixfold coordination order parameter and the self-diffusion coefficients [16]. Using their values of $D_{\mathrm{L}}$ during registering in the above-mentioned analysis yieids a limiting velocily of $3.3 \mu \mathrm{ms}^{-1}$, much lower than that observed in the present study. To recover our limiting velocily, we therefore propose a finite thickness of the interface of $\mathrm{l} \approx 2-5 d_{110}$. Such a result would be consistent also with both recent computer simulations and density functional calculations $[19,24]$.

This andlysis for the first time provides strong evidence for the presence of two limiting processes in the growth of colloidal crystals depending on the respective crystal Iasticc plane. One is due to diffusional transport in the fluid-like ordered melt which is slowed as compared 10 short-time self-diffusion. The other involves a complex three-step mechanism of layer formation, in-layer crystal- 
line ordering and subsequent registering. The first onc is operative at interfaces with only one choice of target places, while the second one oocurs whenever alternative "cgistrations are possible.

\section{Conctustons}

We are still far from a comprehensive underslanding of the kinetics of the first-order freezing transition repeatedly observed also in colloid and interface systems. Performed on the selected case of highly charged latex spheres suspended in aqueous electrolyte, however, this study reporicd some results of broader relevanoe.

The observed phase transition is indeed driven by the potenlial energy difference between solid and melt and it is limited by interfacial dynamics. Thus, our data are excellently described by a Wilson-Frenkel law. A detailed analysis identified two distinct microscopic processes. While the limiting mechanisus may be of difterent nature in other complex huids, the general frame is expected to hold even for systems of complicated internal dynamics.

The suggested derivation of the encrgy density proved successful in our case. This provides a quick but reasonably accurate estimation procedure for the potential energy difference between colloidal melis and the corresponding solids. While the conversion factor $B$ is now only known for boc systerns it is, in principle, also acocssible for other crystal structures.

Future work will therefore involve gradual inclusion of further structural changes and nore sophisticated kinetics. As a first step the growth velocities of systems forming depietion layers could be siudied.

Acknowledgements The authors thank W. Dictcrich, A. Majhofer, and $H$. Löwen for crilical dincussions on the issue of the growth michanisms and the incerfacial thicknisi. R. K'leın is thanked for his critical remarks on the issue of entropic contributions. Financial support from the DFG is gratelully acknowledged.

\section{Reforences}

I. Chen SH, Huang JS, Tartaglia P (1992) Siructure and Dynamios of strongly interacting Culloids and Supramolscular Aggregalc Kluwer, Doorórech1, NATO-ASI 369.39

2. Wilson HA (1990) Philıs Mag 50:238

3. Frenkel J (1932) Phys Z Sowjelunion 1:498

4. Ackerson BJ, Clark NN (1981) Phys Rev Lell 46:123

5. Ackerson BJ (ed.) (1990) Phasc Transitions 21:(2-4)

6. Pusey PN, van Megen W (1989) Nalure 320.340

7. Voeglii LP. Zukoski CE, IV (1991) I Colloid Interlace Sei 14:79

8. Robbins MO, Kremer K, Gresi GS (1988) J Chem Phys 88:3286

9. Palberg T, Mönch W, Bítzer F. Leiderer P. Bellini L. Belloni T, Piaza R (1994) Helvelica Physica Acta 67:225
10. Sirota EB, Ou-Yang HD, Sinha SK. Chaikin PM. Axe JD. Fujii $Y$ (1989) Phys Rev Lall 62:1524

11. Puscy PN in Hansen JP, Levesque D, Zinn-Justin J (eds.) (1989): Liquids, froceing and glass transition", SIs1 summer school in thcoretical physios, Les Houches (F) Elsevitr Amslerdam 1991. pp 763

12. Aastucn DJW. Clark NA, Kotter LK (1986) Phys Rev Lei1 57:1733

13. Aastuen DJW, Clark NA, Swindal JC. Muzny CD in [4], PP 139

14. Gast AP, Monovoukas Y (1991) Naluec 351:552

15. Dhont JKG. Smils C. Lekkerkerker HNW (1992) J Colloid Inlerface Sci 152:386

16. Grier DA. Murray CA (1994) J Chem Phys 100:9088
17. Schälze) K. Ackerson BJ (1993) Phyx Rev E 48:3766

18. Davis KL, Russel WB (1987) Adv Ceram $21: 573$

19. Burke E, Broughton JQ. Glimer GH (1988) J Chem Phys 89:1030

20. Palberg $T$, Wonth $M$, Simon $R$, Leiderer P (1994) Prog Colloid Polym Sci 96:62

21. Palberg T, Koltal J, Bitzer F, Simon R. Wurth M. Leiderer P (1995) J Colloid Inter Sei $168: 85$

22. Biszer F, Palberg T. Löwen H. Simon R. Leiderer P (1994) Phys Rev E 50.2821

23. Palberg T, Hănl W, Witcig U, Versmold H. Würh M. Simruclier E (1992) I Phys Chem 96:8180

24. Nicswand $M$, Majhofer A. Diclerich W (1993) Phys Rev E 48:2521 